\title{
COVID like illness versus COVID-19 (CLIC STUDY) - Clinical profile and outcome
}

\author{
Jeetendra Kumar J Mood', Avinash H Rajanna²,Vaibhav S Bellary³, Gowtham S Gowda4, \\ Yamini Marimuthu ${ }^{5}$
}

${ }^{1}$ Professor, ${ }^{2}$ Assistant Professor, ${ }^{3,4}$ Post Graduate Resident, Department of General Medicine, ${ }^{5}$ Assistant Professor, Department of Community Medicine, ESIC Medical College and PGIMSR, Bengaluru, Karnataka, India

Background: In December 2019, several cases of acute respiratory illness were detected in Wuhan city of China. This SARS-CoV-2 has been rapidly spreading worldwide ever since. SARS-CoV-2 has the potential to damage the vital organs such as lung, heart, liver, and kidney, and infection poses a considerable risk to patients by the high prevalence of pneumonia. Aims and Objectives: The objectives of the study are as follows: (1) To study clinical profile and biochemical markers in SARI patients. (2) To compare the clinical profile and biochemical markers between SARS-CoV-2 positive and negative patients and their outcomes. Materials and Methods: The present study is a hospital-based prospective cross-sectional study conducted on a total of 350 patients (150 SARI + 200 COVID-19) in Bengaluru during the study period from June 2020 to May 2021. Results: Diabetes mellitus was present in $30 \%$ of SARI and $42 \%$ in COVID-19 patients $(P=0.03)$. Leukocytosis (Total Leukocyte Count [TLC] $>11000$ cells $/ \mathrm{mm}^{3}$ ) was more common among SARI patients than COVID-19 patients $(49.3 \%$ vs. $24.3 \%)$. Leukopenia (TLC $<4000$ cells $/ \mathrm{mm}^{3}$ ) was significantly more common in COVID-19 patients than in SARI patients $(10.2 \%$ vs. $3.6 \%$, $\mathrm{P}<0.001)$. Conclusion: COVID-19 infection is more common in patients with comorbidities such as diabetes mellitus and hypertension than SARI. Leukopenia was more common in COVID-19 patients whereas leukocytosis was more common in SARI patients.

Key words: Acute respiratory illness; Diabetes mellitus; Leukopenia; Total leukocyte count

\section{INTRODUCTION}

The World Health Organization (WHO) estimates that acute respiratory infections (ARI) cause annual deaths approaching 4 million, at a rate of more than 60 deaths $/ 100,000$ populations. Viruses are responsible for $30-70 \%$ of ARI where respiratory syncytial virus, influenza virus, parainfluenza virus, human bocavirus, human meta pneumovirus, adenovirus, rhinovirus, enterovirus, and coronaviruses account for the majority of these cases. ${ }^{1,2}$

Although likely to have been started as a zoonotic transmission in the large sea food market of Wuhan, human-to-human transmission through droplets and contact with fomites has since been established to be the modus operandi of the virus spread. ${ }^{3}$ COVID-19 infection encompasses a variety of presentations which can be asymptomatic infection, fever, cough, fatigue, shortness of breath, pneumonia, and other respiratory tract symptoms and in many cases progresses to severe respiratory failure and death. ${ }^{4,5}$

Coronaviruses are non-segmented positive-stranded RNA viruses with a roughly $30 \mathrm{~kb}$ genome surrounded by a protein envelope. Most coronaviruses cause diseases in their particular host species. ${ }^{6}$ Those that can infect humans through cross-species transmission have become an important threat to public health. Two serious coronavirus disease outbreaks have happened in the past two decades: Severe acute respiratory syndrome (SARS) in $2003^{7}$ and Middle East respiratory syndrome ${ }^{8}$ in 2012. 
Since December 2019, SARS coronavirus 2 (SARS-CoV-2) has been recognized as the causal factor in a series of severe cases of pneumonia originating in Wuhan in Hubei Province, China.?

This disease has been named coronavirus disease 2019 (COVID-19) by the WHO. SARS-CoV-2 has been shown to cause disease through a mechanism analogous to the SARS coronavirus, with potential damage to vital organs such as lung, heart, liver, and kidney, and the infection poses a considerable risk to patients by the high prevalence of pneumonia ${ }^{10}$

In India, thousands of cases of COVID-19 are being reported from all the states with Maharashtra being the most affected state in the country. ${ }^{11}$ In India, from January 3, 2020, to 4:07 pm CEST, June 6, 2021, there have been 28,809,339 confirmed cases of COVID-19 with 346,759 deaths, reported to the WHO. ${ }^{12}$

Patients have been presenting with features of severe acute respiratory illness and then eventually getting diagnosed with either COVID-19 positive or negative status. Any suspect case who is RT-PCR for COVID-19 negative but clinico-radiological evidence and laboratory values are suggestive of COVID-19 disease as per criteria: a) Clinical features suggestive of COVID-19, b) radiological evidence - CT Thorax/Chest X-Ray, c) blood investigations showing lymphocytopenia, CRP >100 milligram/liter, Ferritin $>300$ microgram/liter, Serum Lactate Dehydrogenase $(\mathrm{LDH})>450$, and D-dimer $>1000$ nanogram/milliliter, and d) nasal and throat swab RT-PCR for COVID-19 negative are to be treated as COVID like illness, that is, $\mathrm{a}+\mathrm{b}+\mathrm{c}+\mathrm{d}=$ COVID like illness as per guidelines issued by the Department of Health and Family Welfare of India dated October 15, 2020. ${ }^{13}$

In the present study, we aim to study the clinical profile and biochemical markers in SARI patients and also to compare the clinical profile and biochemical markers between SARS$\mathrm{CoV}-2$ positive and negative patients and also their outcomes.

\section{Aims and objectives}

The objectives of the study are as follows:

1. To study clinical profile and biochemical markers in SARI patients.

2. To compare the clinical profile and biochemical markers between SARS-Cov-2 positive and negative patients and their outcomes.

\section{MATERIALS AND METHODS}

The present study was conducted in a hospital in Bengaluru, Karnataka, India. Ethical clearance for the study was obtained from the Institutional Ethics Committee (reference no: 532/L/11/12/Ethics/ESICMC\&PGIMSR/ Estt.Vol.IV) in accordance with the guidelines issued by the Indian Council of Medical Research.

Data were collected from a total of 350 patients presenting to the Department of General Medicine Triage and COVID Ward/ICU in ESIC-PGIMSR, Bengaluru from June 2020 to May 2021, fulfilling the inclusion criteria.

After obtaining approval and clearance from the institutional ethics committee, the patients fulfilling the inclusion criteria were enrolled for the study after obtaining informed consent. Case record form with follow-up chart was used to record the duration of disease, history of treatment, and complications. COVID-19 infection was diagnosed by RT-PCR and rapid antigen test (RAT) technique. Patients underwent biochemical investigations which included complete blood count, liver function test, renal function test, serum electrolytes, serology, CRP, LDH, D-dimer, ABG, and chest X-ray. Comorbid conditions such as metabolic disorders, endocrine disorders, renal disorders, cardiac disorders, and respiratory disorders were confirmed with medical history.

\section{Inclusion criteria}

The following criteria were included in the study

1. Patient/attender willing to give informed consent

2. Patients of either sex with age more than 18 years

3. Patients admitted in ESI triage and diagnosed as SARI

4. Patients who test positive for COVID-19 either by RT-PCR/RAT for COVID-19.

\section{Exclusion criteria}

The following criteria were excluded from the study

1. Patient not willing to give informed consent

2. Age $<18$ years.

With the expected proportion of SARI patients who presented with ARI as 40.6 (ref), a relative precision of $20 \%$ and $95 \%$ confidence level, the sample size was calculated to be 141 using OpenEpi version 3. The sample size was then rounded off to 150 SARI patients. Since there were no literatures comparing the clinical profile of SARI and COVID-19 patients, we took 200 confirmed COVID-19 patients admitted during the study period and compared with the SARI patients as per study conducted by Aggarwal et al. ${ }^{14}$

Data were collected from case files of patients admitted to the Triage/COVID ward and ICU. History, laboratory investigations (TLC, N: L, D-dimer, CRP, and LDH), and radiological investigations were collected. Patients were divided into two groups, one who tested positive for COVID-19 infection $(\mathrm{n}=200)$ and the other who did 
not $(\mathrm{n}=150)$. SARI cases with RT-PCR negative have been included under the term COVID like illness in our study. The patients in both the groups were monitored and the clinical profile and laboratory investigations were measured either as improvement (clinical improvement, decreasing trend of inflammatory markers, and discharge) or deterioration (clinically worsening, increasing trend of inflammatory markers, and death) of the patient.

\section{Method of statistical analysis}

Data were entered into a Microsoft Excel datasheet and were analyzed using SPSS 22 version software. Categorical data were represented in the form of frequencies and proportions. Chi-square test was used as a test of significance for qualitative data. Continuous data were represented as mean and standard deviation. $\mathrm{P}<0.05$ was considered as statistically significant.

\section{RESULTS}

A total of 150 SARI patients and 200 COVID-19 patients were included in our study.

\section{Demographic data}

About $52 \%$ of SARI patients were in the age group of $31-60$ years whereas $60 \%$ of COVID-19 patients were in the age group of $31-60$, as shown in Table 1.

Of the 150 SARI patients, $54 \%$ were male and, of the 200 COVID-19 patients, $66.7 \%$ were male, as represented by Figure 1.

\section{Comorbidities}

As outlined in Table 2, diabetes mellitus was present in $30 \%$ of SARI whereas $42 \%$ in COVID-19 patients and this difference is statistically significant $(\mathrm{p}=0.03)$. About $28.7 \%$ of SARI patients and $38.7 \%$ of COVID-19 patients had hypertension. About $13 \%$ of SARI patients and $12 \%$ of COVID-19 patients had ischemic heart disease which was statistically insignificant $(\mathrm{p}=0.73)$.

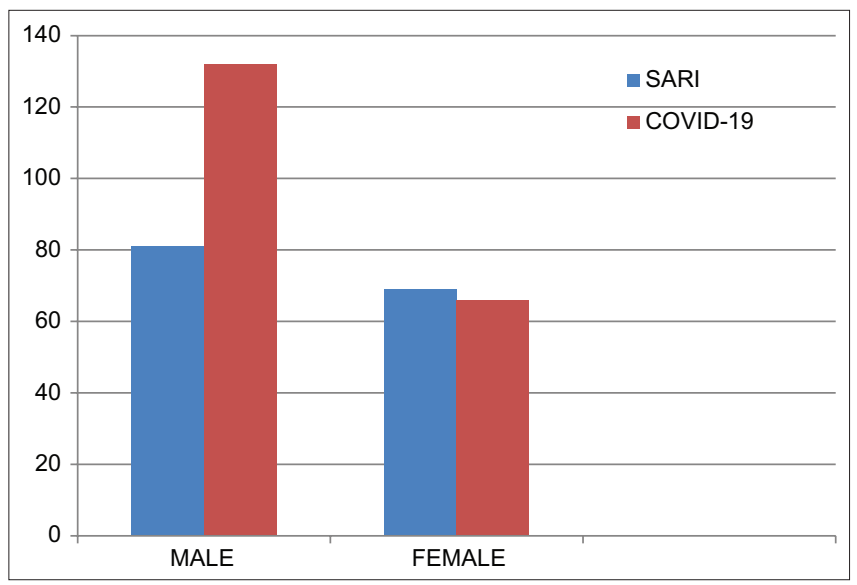

Figure 1: Sex distribution
In Table 3, we see that a total leukocyte count (TLC) of more than 11000 cells $/ \mathrm{mm}^{3}$ was present in $49.3 \%$ of SARI patients and $24.3 \%$ in COVID-19 patients. This difference was statistically significant with P value of 0.001 . Moreover, TLC $<4000$ cells $/ \mathrm{mm}^{3}$ was present in $10.2 \%$ of COVID-19 patients and $3.6 \%$ of SARI patients. This suggests that leucopenia is more in COVID-19 patients and leukocytosis is more in SARI patients.

Furthermore, the $\mathrm{D}$-dimer was increased in $68.2 \%$ of SARI patients when compared to $48.9 \%$ of COVID-19 patients. This difference is statistically significant with $\mathrm{P}$ value of 0.031 .

Other inflammatory markers such as C-Reactive Protein (CRP), Lactate Dehydrogenase (LDH), and NeutrophilLymphocyte Ratio (NLR) when compared in both the groups were not statistically significant.

Outcome: As pointed out by Table 4, there was no statistical significance seen in the outcome of the patients from both the COVID-19 and SARI groups.

\section{DISCUSSION}

The SARS-CoV-2 virus as per the International Committee on Taxonomy of Viruses or COVID-19 as we now know has been rapidly spreading worldwide thereafter. COVID-19 was declared a pandemic by the WHO on March 11, 2020.

In the present study, we compare the clinical profile and outcomes of the patients of COVID-19 and SARI in the form improved (clinical improvement, decreasing trend of inflammatory markers, and discharge) or deteriorated (clinically worsening, increasing trend of inflammatory markers, and death of the patient).

\begin{tabular}{lcccc}
\multicolumn{4}{l}{ Table 1: Age distribution } \\
\hline Factor & Age Groups & SARI & COVID-19 & P-value \\
\hline Number & & 150 & 200 & \\
Age & $0-30$ & $6(4.0 \%)$ & $10(5.1 \%)$ & 0.22 \\
& $31-60$ & $78(52.0 \%)$ & $119(60.1 \%)$ & \\
& $61-90$ & $66(44.0 \%)$ & $69(34.8 \%)$ & \\
\hline
\end{tabular}

\begin{tabular}{lccc}
\multicolumn{3}{l}{ Table 2: Comorbidities } & \\
\hline SARI & & COVID-19 & P-value \\
\hline HTN & $107(71.3 \%)$ & $92(61.3 \%)$ & 0.067 \\
No & $43(28.7 \%)$ & $58(38.7 \%)$ & \\
Yes & & & \\
DM & $105(70.0 \%)$ & $87(58.0 \%)$ & 0.030 \\
No & $45(30.0 \%)$ & $63(42.0 \%)$ & \\
Yes & & & \\
IHD & $130(86.7 \%)$ & $132(88.0 \%)$ & 0.73 \\
No & $20(13.3 \%)$ & $18(12.0 \%)$ & \\
Yes & & & \\
\hline
\end{tabular}




\begin{tabular}{|c|c|c|c|c|}
\hline Factor & Levels & SARI & COVID-19 & P-value \\
\hline Number & & 150 & 200 & \\
\hline \multirow[t]{3}{*}{ Total Leukocyte Count } & $0-4000$ & $5(3.6 \%)$ & $18(10.2 \%)$ & $<0.001$ \\
\hline & $4001-11000$ & $65(47.1 \%)$ & $116(65.5 \%)$ & \\
\hline & $>11000$ & $68(49.3 \%)$ & $43(24.3 \%)$ & \\
\hline \multirow[t]{3}{*}{ Neutrophil: Lymphocyte Ratio } & $0-3$ & $21(15.7 \%)$ & 37 (21.4\%) & 0.071 \\
\hline & $3.1-9$ & 57 (42.5\%) & 85 (49.1\%) & \\
\hline & $>9$ & 56 (41.8\%) & $51(29.5 \%)$ & \\
\hline \multirow[t]{3}{*}{ Lactate Dehydrogenase } & $0-140$ & $1(1.3 \%)$ & $10(8.4 \%)$ & 0.12 \\
\hline & $141-280$ & $22(29.3 \%)$ & 33 (27.7\%) & \\
\hline & $\geq 281$ & $52(69.3 \%)$ & $76(63.9 \%)$ & \\
\hline \multirow[t]{3}{*}{ D-dimer } & $0-0.599$ & $21(31.8 \%)$ & $69(50.4 \%)$ & 0.031 \\
\hline & $0.6-10$ & $45(68.2 \%)$ & $67(48.9 \%)$ & \\
\hline & & $0(0.0 \%)$ & $1(0.7 \%)$ & \\
\hline C-Reactive Protein & & $2.4(0.7,2.4)$ & $1.4(0.7,2.4)$ & 0.37 \\
\hline
\end{tabular}

\section{Table 4: Outcomes of the patients}

\begin{tabular}{lccc}
\hline Group & Discharged & Expired & Total \\
\hline SARI & 136 & 14 & 150 \\
& $90.67 \%$ & $9.33 \%$ & $100 \%$ \\
COVID-19 & 180 & 20 & 200 \\
& $90 \%$ & $10 \%$ & $100 \%$ \\
TOTAL & 316 & 34 & 350 \\
& $90.29 \%$ & $9.71 \%$ & $100 \%$ \\
& Pearson Chi-square & $\mathrm{P}=0.835$ \\
& \multicolumn{3}{c}{$(1)=0.0434$} \\
\hline
\end{tabular}

In the present study, $52 \%$ of SARI patients were in the age group of 31-60 years whereas 60\% of COVID-19 patients were in the age group of 31-60. Of the 150 SARI patients, $54 \%$ were male and, of the 200 COVID-19 patients, $66.7 \%$ were male.

Diabetes mellitus was found to be present in $30 \%$ of SARI and $42 \%$ in COVID-19 patients and this difference is statistically significant with $\mathrm{p}$ value of 0.03 . About $28.7 \%$ of SARI patients and $38.7 \%$ of COVID-19 patients had hypertension, and $13 \%$ of SARI patients and $12 \%$ of COVID-19 patients had ischemic heart disease which was statistically insignificant. A study conducted by Huang et al., concluded that diabetes mellitus was associated with mortality, severe COVID-19, ARDS, and disease progression in patients with COVID-19. ${ }^{15}$

Aggarwal et al., showed that $68.8 \%$ patients had one or more comorbidities of which diabetes mellitus prevailed in $50 \%$, hypertension in $34.4 \%$, and chronic obstructive airway disease in $15.6 \%$ of the COVID-19 patients compared to the SARI patients. ${ }^{14}$

In our study, TLC more than 11000 cells $/ \mathrm{mm}^{3}$ was present in $49.3 \%$ of SARI patients and $24.3 \%$ in COVID-19 patients. This difference was statistically significant with $\mathrm{P}$ value of 0.001 . Moreover, TLC $<4000$ cells $/ \mathrm{mm}^{3}$ was present in $10.2 \%$ of COVID-19 patients and $3.6 \%$ of SARI patients. This suggests that leukopenia is more common in COVID-19 patients and leukocytosis in SARI patients.

Furthermore, the D-dimer was increased in $68.2 \%$ of SARI patients when compared to $48.9 \%$ of COVID-19 patients. This difference is statistically significant with $\mathrm{P}$ value of 0.031 .

Other inflammatory markers such as CRP, LDH, and NLR when compared in both the groups were not statistically significant.

A study conducted by Kumar et al., concluded that Remdesivir (RDV) only caused improvement in certain inflammatory markers such as D-dimer and LDH. ${ }^{16}$ Furthermore, RDV was only found to be beneficial when administered within the first 10 days of symptom onset as per the study conducted by Avinash et al. ${ }^{17} \mathrm{~A}$ variety of therapeutic approaches including novel antivirals, immune-modulators, and combination approaches have to be studied in detail to continue to improve outcomes in patients with COVID-19 as well as SARI cases to successfully help tackle this pandemic.

\section{Limitations of the study}

The study is, however, not without limitations. The sample size was small and also it was a single center study.

\section{CONCLUSION}

COVID-19 infection is more common in patients with comorbidities such as diabetes mellitus and hypertension than SARI. Leukopenia was more common in COVID-19 patients and leukocytosis was more common in SARI patients.

\section{ACKNOWLEDGMENT}

The authors would like to thank the Department of General Medicine for their whole hearted support. 


\section{REFERENCES}

1. Murray C, Lopez A, Mathers C and Stein C. The Global Burden of Disease 2000 Project: Aims, Methods, and Data Sources. Global Programme on Evidence for Health Policy. Geneva: World Health Organization; 2001. Available from: http://www. who.int/healthinfo/paper36.pdf [Last accessed on 2021 Oct 10].

2. Tregoning JS and Schwarze J. Respiratory viral infections in infants: Causes, clinical symptoms, virology, and immunology. Clin Microbiol Rev. 2010;23(1):74-98.

https://doi.org/10.1128/CMR.00032-09

3. Zhou F, Yu T, Du R, Fan G, Liu Y, Liu Z, et al. Clinical course and risk factors for mortality of adult inpatients with COVID-19 in Wuhan, China: A retrospective cohort study. Lancet. 2020;395(10229):1054-1062.

https://doi.org/10.1016/S0140-6736(20)30566-3

4. Chen N, Zhou M, Dong X, Qu J, Gong F, Han Y, et al. Epidemiological and clinical characteristics of 99 cases of 2019 novel Coronavirus pneumonia in Wuhan, China: A descriptive study. Lancet. 2020;395(10223):507-513.

https://doi.org/10.1016/S0140-6736(20)30211-7

5. Wang D, Hu B, Hu C, Zhu F, Liu X, Zhang J, et al. Clinical characteristics of 138 hospitalized patients with 2019 novel Coronavirus-infected pneumonia in Wuhan, China. JAMA. 2020;323(11):1061-1069.

https://doi.org/10.1001/jama.2020.1585

6. Shi $Z$ and $\mathrm{Hu} Z$. A review of studies on animal reservoirs of the SARS Coronavirus. Virus Res. 2008;133(1):74-87.

https://doi.org/10.1016/j.virusres.2007.03.012

7. Donnelly CA, Ghani AC, Leung GM, Hedley AJ, Fraser C, Riley S, et al. Epidemiological determinants of spread of causal agent of severe acute respiratory syndrome in Hong Kong. Lancet. 2003;361(9371):1761-166.

https://doi.org/10.1016/S0140-6736(03)13410-1

8. Cauchemez S, Fraser C, van Kerkhove MD, Donnelly CA, Riley S, Rambaut A, et al. Middle East respiratory syndrome coronavirus: Quantification of the extent of the epidemic, surveillance biases, and transmissibility. Lancet Infect Dis. 2014;14(1):50-56.

https://doi.org/10.1016/S1473-3099(13)70304-9
9. Wu P, Hao X, Lau EH, Leung KS, Wu JT, Cowling BJ, et al. Realtime tentative assessment of the epidemiological characteristics of novel Coronavirus infections in Wuhan, China, as at 22 January 2020. Euro Surveill. 2020;25(3):2000044.

https://doi.org/10.2807/1560-7917.ES.2020.25.3.2000044

10. Hamming I, Timens W, Bulthuis ML, Lely AT, Navis G and van Goor $H$. Tissue distribution of ACE2 protein, the functional receptor for SARS Coronavirus: A first step in understanding SARS pathogenesis. J Pathol. 2004;203(2):631-637. https://doi.org/10.10.1002/path.1570

11. Ministry of Health and Family Welfare, COVID-19-India; 2020. Available from: https://www.mohfw.gov.in [Last accessed on 2021 Oct 10].

12. World Health Organization. India: WHO Coronavirus Disease (COVID-19) Dashboard with Vaccination Data. Geneva: World Health Organization; 2021. Available from: https://www. covid19.who.int/region/searo/country/in [Last accessed on 2021 Jun 07].

13. Syndromic Approach to COVID-19 Disease. 15AD. Bangalore: Government of Karnataka; 2020.

14. Aggarwal A, Shrivastava A, Kumar A and ALi A. Available from: https://www.japi.org/w2f4d434/clinical-and-epidemiologicalfeatures-of-sars-cov-2-patients-in-sari-ward-of-a-tertiary-carecentre-in-new-delhi [Last accessed on 2021 Aug 24].

15. Huang I, Lim MA and Pranata R. Diabetes mellitus is associated with increased mortality and severity of disease in COVID-19 pneumonia-a systematic review, meta-analysis, and metaregression. Diabetes Metab Syndr. 2020;14(4):395-403. https://doi.org/10.1016/j.dsx.2020.04.018

16. Kumar JM, Vaibhav SB, Avinash HR and Pratheek P. Remdesivir versus standard of care in moderate to severe COVID-19 patients: A retrospective study. J Clin Diagn Res. 2021;15(4):OC14-OC17. Available from: https://www.jcdr.net/ article_fulltext.asp?id=14767

17. Avinash HR, Satyanarayana N, Vaibhav SB and Pratheek P. To compare the outcome of patients who are receiving remdesivir within 10 days of symptom onset to those receiving remdesivir after 10 days of symptom onset. Int $\mathrm{J}$ Innov Res Med Sci. 2021;6(2):158-161. https://doi.org/10.23958/ijirms/vol06-i02/1066

\footnotetext{
Authors contribution:

JKJM- Project Administration, Supervision, Resources, and Validation; AHR- Conceptualization, Data Curation, Methodology, and Validation;

VSB- Conceptualization, Data Curation, Investigation, Software, Writing - original draft, and Writing - review and editing; GSG- Project Administration, Formal

Analysis, and Visualization; YM- Methodology, Data Interpretation, and Software

Work attributed to:

ESIC Medical College and PGIMSR, Rajajinagar, Bengaluru - 560 010, Karnataka, India

Orcid ID:

Dr. Jeetendra Kumar J Mood - (1) https://orcid.org/0000-0002-8030-8448

Dr. Avinash H Rajanna - (1) https://orcid.org/0000-0001-6484-5190

Dr. Vaibhav S Bellary - (1) https://orcid.org/0000-0003-2699-988X

Dr. Gowtham S Gowda - (1) https://orcid.org/0000-0003-0750-2364

Dr. Yamini Marimuthu - (i) https://orcid.org/0000-0003-0599-2945

Source of Support: Nil, Conflict of Interest: None declared.
} 